\title{
EDITORIAL
}

\section{Future therapeutics through synthetic biology}

Synthetic biology has positioned itself capable of doing what synthetic organic chemistry did to change the scientific landscape a century ago (Yeh and Lim, 2007; Smansk et al., 2016). Organic synthesis, in spite of many notable advances, especially in the synthesis of prevalent Natural Product (NP) backbones containing fiveand six-membered ring compounds (Nicolaou, 2014; Piers and Karunaratne, 1983; Posner, 1986), has not become the panacea for the production of NPs for the pharmaceutical industry. NPs, which are specialized metabolites produced by plants, animals and microorganisms, with diverse chemical structures and biological activities are valuable in a clinical setting, with half of small molecule drugs approved during the past three decades being derived from NPs (Newman and Cragg, 2012). However, the diversity within biologically relevant 'chemical space' is more important than the size of any library of synthetic compounds. NP libraries show a wide range of pharmacophores with diverse stereochemistry, and these attributes may be more important in being able to deliver "hits", even against biological targets. While NPs are not only used in the treatment of infection and cancer and as immunosuppressive agents, they have also made it into commercial products as antivirals, anthelmintics, enzyme inhibitors and nutraceuticals. In many NP-producing organisms, genes essential for regulation, biosynthesis, export and self-resistance are colocalized in the genome in compact 'biosynthetic gene clusters' (BGCs).

Because synthetic strategies in making key natural products have been relatively lowthroughput methods at best, NPs appear larger than life, an inexhaustible resource. In the coming decades, advances in technology are poised to narrow this gap allowing for a more systematic approach to global NP production. Ever improving bioinformatics and ready availability of sequenced genomes, make it possible to assess the potential number of undiscovered natural products. For example, in Streptomyces alone, there may be about 150,000 such NPs, of which $<5 \%$ are known. In addition, bioinformatics have laid bare hundreds of genomes in many genera giving access to 100,000 s of NPs.

Engineering NP biosynthesis chooses tools from a variety of subfields in genetic engineering and chemistry. Firstly, genetic function must be separated into its components such as promoters that generate precise levels of gene expression, creating large libraries of well understood parts and the development of biophysical and bioinformatics models to predict the behaviour of components. Component libraries for different organisms will assist the transfer of BGCs between hosts.

Secondly, synthetic biology will benefit from new developments in DNA synthesis capacity. Now it is possible to routinely synthesize the $20 \mathrm{~kb}-100 \mathrm{~kb}$ needed for a large gene cluster, the assembly of which has been enabled by rapid construction of different component combinations. New computer aided design methods have enhanced the means of designing genetic systems and locating system errors. Today, genetic circuits have been made to function as logic, timers, switches and oscillators. Sensors have also been developed that respond to many inducible inputs and that know metabolite levels. NP pathways can use these advances to control the timing of expression of different genes or to initiate responses to toxic intermediates. 
The recent upsurge in DNA sequencing methods have shown that BGCs for NP production are more extensive than previously conceived, and the ability to write DNA roadmaps into synthetic pathways is now gathering momentum. New approaches for harvesting NPs from genomic sequences are required as never before to revive declining drug discovery avenues, especially considering the alarming crisis of antibiotic resistance.

In Sri Lanka, 25\% of its higher plants are endemic and has shown significant biological activity (Hewage et al., 1997). Lower plants have shown no less chemical diversity (Karunaratne et al., 2005). However, thus far, these favorable factors have not yielded commercially useful NPs in Sri Lanka. The NPs present in nature is one of our most valuable natural resources, and synthetic biology will allow ways to investigate its scope and diversity in the future.

\section{REFERENCES}

Hewage, C.M., Bandara, K.A.N.P., Karunaratne, V., Bandara, B.M.R. and Wijesundara, D.S.A. (1997). Insecticidal activity of some medicinal plants of Sri Lanka. Journal of National Science Council 25(3): 141-150.
Karunaratne, V., Bombuwela, K., Kathirgamanathar, S. and Thadhani, V.M. (2005). Lichens: a chemically important biota. Journal of National Science Foundation 33(3): 169-86.

Newman, D. J. and Cragg G. M. (2012). Natural products as sources of new drugs over the 30 years from 1981 to 2010. Journal of Natural Products 75: 311-335.

Nicolaou, K. C. (2014). Organic synthesis: the art and science of replicating the molecules of living nature and creating others like them in the laboratory. Proceedings of The Royal Society A 470(2163): 20130690.

Piers, E. and Karunaratne, V. (1983). Conjugate addition of Lithium Phenylthio- and cyano-[2-(4chlorobut-l-enyl) cuprate to cyclic enones. An efficient methylenecylcopentane annulation process. Journal of Chemical Society and Chemical Communications: 935-936.

Posner, G.H. (1986). Multicomponent one-pot annulations forming 3 to 6 bonds. Chemical Reviews 86(5): 831-844.

Smansk, M. J., Zhou, H., Claesen, J., Shen, B., Fischbach, M. and Voigt, C. A. (2016). Synthetic biology to access and expand nature's chemical diversity. Nature Reviews Microbiology 14(3): 135-149.

Yeh, B. J. and Lim, W. A. (2007). Synthetic biology: lessons from the history of synthetic organic chemistry. Natural Chemical Biology 3(9): 521525.

Veranja Karunaratne 